\title{
Power dependence of phase noise in microwave kinetic inductance detectors
}

Jiansong Gao, Benjamin Mazin, Miguel Daal, Peter Day, Henry LeDuc, et al.

Jiansong Gao, Benjamin Mazin, Miguel Daal, Peter Day, Henry LeDuc, Jonas Zmuidzinas, "Power dependence of phase noise in microwave kinetic inductance detectors," Proc. SPIE 6275, Millimeter and Submillimeter Detectors and Instrumentation for Astronomy III, 627509 (27 June 2006); doi: $10.1117 / 12.672590$

Event: SPIE Astronomical Telescopes + Instrumentation, 2006, Orlando, Florida, United States 


\title{
Power dependence of phase noise in microwave kinetic inductance detectors
}

\author{
Jiansong Gao ${ }^{a}$, Benjamin Mazin ${ }^{b}$, Miguel Daal $^{c}$, Peter Day $^{b}$ Henry LeDuc $^{b}$, and Jonas \\ Zmuidzinas $^{a}$ \\ ${ }^{a}$ California Institute of Technology, Pasadena, CA 91125 \\ ${ }^{b}$ Jet Propulsion Laboratory, Pasadena, CA 91109 \\ ${ }^{c}$ University of California at Berkeley, Berkeley, CA 94720
}

\begin{abstract}
Excess phase noise has been observed in microwave kinetic inductance detectors (MKIDs) which prevents the noise-equivalent power (NEP) of current detectors from reaching theoretical limits. One characteristic of this excess noise is its dependence on the power of the readout signal: the phase noise decreases as the readout power increases. We investigated this power dependence in a variety of devices, varying the substrate (silicon and sapphire), superconductor (aluminum and niobium) and resonator parameters (resonant frequency, quality factor and resonator geometry). We find that the phase noise has a power law dependence on the readout power, and that the exponent is $-1 / 2$ in all our devices. We suggest that this phase noise is caused by coupling between the high- $Q$ microwave resonator that forms the sensitive element of the MKID and two-level systems associated with disorder in the dielectric material of the resonator. The physical situation is analogous to the resonance fluorescence in quantum optics, and we are investigating the application of resonance fluorescence theory to MKID phase noise.
\end{abstract}

Keywords: kinetic inductance detector, phase noise, two level system, resonance fluorescence

\section{INTRODUCTION}

The microwave kinetic inductance detector (MKID) is an emerging superconducting photon detector technology that has a wide range of potential applications from millimeter-wave frequencies to X-rays. ${ }^{1-10}$ MKIDs are distinct from other superconducting detectors in two important aspects. First, MKIDs detect a change in the kinetic inductance, as compared to a change in the resistance in transition edge sensor (TES) ${ }^{11}$ or a change in the tunneling current in superconducting tunnel junction detector (STJ). ${ }^{12}$ Second, MKIDs use microwave resonator circuit for signal readout, as compared to SQUID readout for TES devices or JFET readout for STJs. These detector concepts make MKIDs simple to multiplex and easy to fabricate.

Although the noise performance of MKIDs is already acceptable for ground-based astronomy, excess noise is observed that prevents current detectors from reaching the theoretical limit. This excess noise appears purely as phase noise and strongly depends on the substrate material and the power of the readout signal. We propose that the excess phase noise is due to two level systems (TLSs) in the dielectric material of the resonator.

This paper is divided into four sections. Sections 2 and 3 give brief introductions to the device physics and the experimental setup. In Section 4, experimental observations of this excess phase noise, in particular, its power and material dependance, are presented. In Section 5, the two-level-system model and the resonance fluorescence theory are introduced which may provide an explanation for the excess phase noise.

Send correspondence to Jiansong Gao. E-mail: jiansong@caltech.edu, Telephone: 6263952647

Millimeter and Submillimeter Detectors and Instrumentation for Astronomy III, edited by Jonas Zmuidzinas, Wayne S. Holland, Stafford Withington, William D. Duncan, Proc. of SPIE Vol. 6275, 627509, (2006) · 0277-786X/06/\$15 - doi: 10.1117/12.672590

Proc. of SPIE Vol. 6275 627509-1 

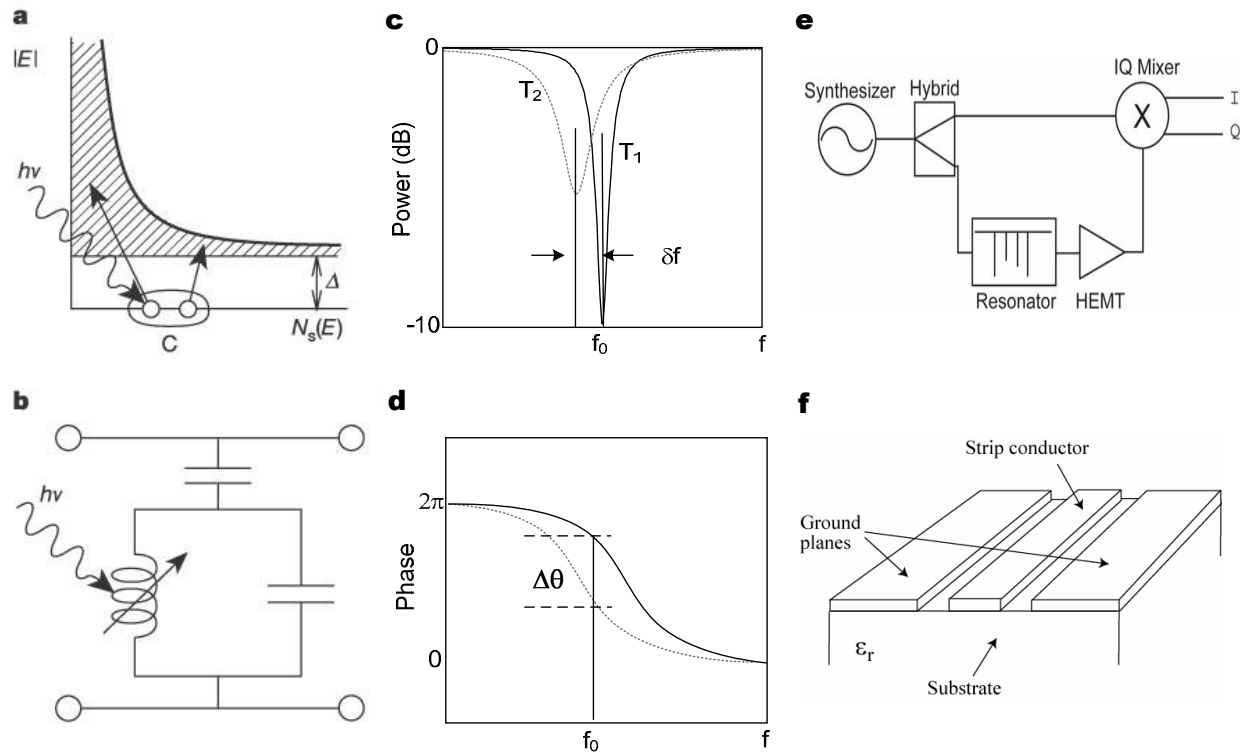

Figure 1. Principle of operation of MKID. a) Input energy $(E>2 \Delta, \Delta$ is the gap energy of the superconductor $)$ breaks Cooper pairs and creates quasiparticles. b) MKID as an inductive element in LC resonator. c) Narrow dip at resonance frequency in transmission moves when kinetic inductance changes. d) The circuit goes off-resonance and the output signal acquires a phase shift. e) Diagram of the readout system. f) Coplanar waveguide (CPW) geometry of the device.

\section{DEVICE PHYSICS}

The device physics of an MKID is straightforward, ${ }^{1}$ and is explained in detail in Figure 1. The sensor of MKID is a strip of superconducting film patterned into a transmission line resonator operating at a temperature far below the transition temperature $\left(T \ll T_{c}\right)$. Incident photon breaks Cooper pairs in the strip (Figure 1(a)), generating quasiparticles (QPs), which changes the microwave inductance (Figure 1(b)) and resistance, and results in a change in the resonance frequency $f_{0}$ and the quality factor $Q$ of the resonator (Figure 1(c)). If a microwave signal tuned to the resonance frequency is applied, a phase and amplitude change can be detected in the reflected or transmitted wave from the resonator (Figure 1(d)). One of the most attractive aspects of this scheme is that large detector arrays may be read out using frequency domain multiplexing. This can be achieved by sending a comb of microwave frequencies matching each resonator, amplifying the transmitted signal with a single cryogenic HEMT amplifier, and demultiplexing at room temperature. ${ }^{7}$ Because MKIDs are essentially single-layer superconducting strips with their lengths setting the resonance frequencies and no additional circuitry is needed, they are easy to fabricate.

\section{EXPERIMENTAL SETUP}

\subsection{Readout system}

Figure 1(e) is a diagram of the readout system which implements the homodyne mixing scheme to measure the phase and amplitude of a microwave resonator. ${ }^{13}$ A microwave frequency synthesizer creates a signal which is split into two by a splitter. One signal goes into the LO port of an IQ mixer and the other goes through the cryostat, resonator, and amplifiers and into the RF port of the IQ mixer. The IQ mixer contains two mixers, one of which includes a 90 degree phase shift before its LO. With the amplitude of LO signal fixed, the output voltage of the IQ mixer (denoted by a complex number $Z$ ) gives the complex amplitude of the RF signal $\left(A_{R F}\right)$

$$
Z=V_{I}+i V_{Q} \propto A_{R F} .
$$

The $I$ and $Q$ channels are amplified, filtered, and digitized for further analysis. By measuring $I$ and $Q$ while we sweep the microwave frequency around the resonant frequency of a resonator, we can determine the quality factor $(Q)$ and resonant frequency $\left(f_{0}\right)$ by fitting the data to a resonator model. 


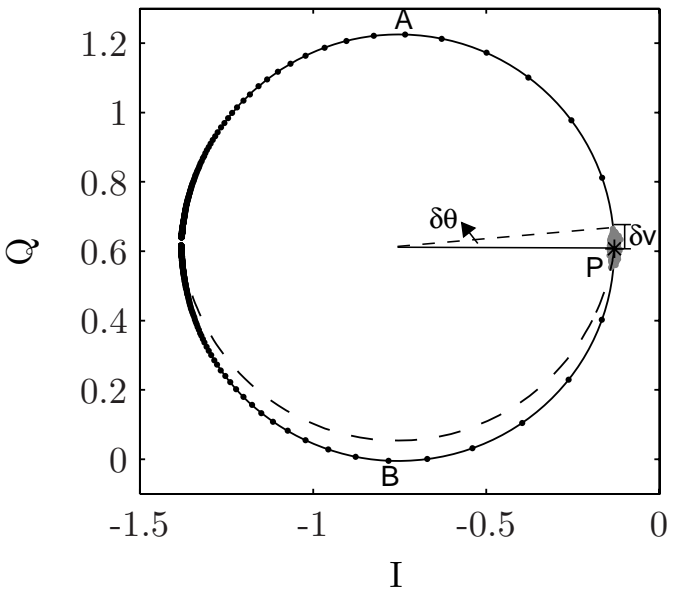

(a)

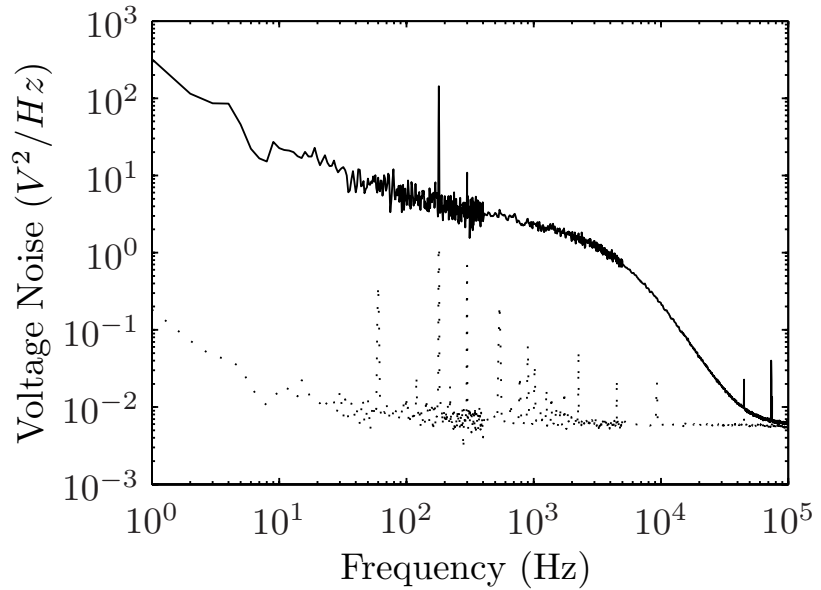

(b)

Figure 2. Voltage noise and associated spectra. (a) Resonance circle (solid line), quasi-particle trajectory (dashed line) and noise in the $I Q$ plane. The data is from a Nb resonator $\left(f_{0}=4.3 \mathrm{GHz}, Q \sim 3 \times 10^{5}\right)$ on Si substrate at $120 \mathrm{mK}$. $P$ indicates the resonance frequency. The shaded area shows the noise blob which is generated by overlapping one second of noise data (sampling rate $250 \mathrm{kHz}$ ). Point $A$ and $B$ are at half bandwidth $\left(f_{0} / 2 Q\right)$ away from the resonance frequency. The quasi-particle trajectory (dashed line) is calculated from $\sigma_{1}$ and $\sigma_{2}$, the complex conductivity, using Mattis-Bardeen theory. ${ }^{14}$ (b) Spectra of voltage noise in the phase (solid line) and amplitude (dashed line) directions.

\subsection{Device Fabrication}

MKID device is usually a quarter-wave transmission line resonator with one end shorted to the ground. For the advantages of low radiation loss and easy fabrication, we use coplanar waveguide (CPW) geometry (shown in Figure 1(f)). A superconducting thin film is deposited on some substrate and patterned using photolithography into a center strip and two gaps. Each device discussed in this paper has a $3 \mu m$ wide center strip and $2 \mu m$ wide gaps, while the thickness, type of superconductor and substrate varies.

\section{EXPERIMENTAL RESULTS}

\subsection{Excess noise in phase direction}

Figure 2(a) shows the resonance circle in the $I Q$ plane as a function of frequency measured from a $\mathrm{Nb}$ resonator on Si substrate. The resonance frequency is indicated by point $P$. Even without incident photon, point $P$ will jitter randomly around its mean position due to noise. To fully examine the noise, we measure the auto-power spectrum $S_{I I}$ and $S_{Q Q}$, and the cross-power spectrum $S_{I Q}$ of the $I$ and $Q$ signals by an audio spectrum analyzer. These noise spectra are then projected into the phase direction (the tangential direction of the resonance circle) and the amplitude direction (the radial direction of the resonance circle).

The resulting two spectra are shown in Figure 2(b). In the amplitude direction, the noise is flat and matches both the noise spectrum measured at off-resonance frequencies and the estimated noise temperature ( $\sim 5 \mathrm{~K})$ of our HEMT amplifier. Therefore, no excess noise is measured in the amplitude direction; rather, the amplitude noise is limited by the HEMT amplifier. In the phase direction, we find an excess noise well above the HEMT noise, with a $1 / f$ profile at low frequency (below $10 \mathrm{~Hz})$ and a roll-off at the resonator's bandwidth $f_{0} / 2 Q(\sim 7$ $\mathrm{kHz})$.

The observed excess phase noise is much higher than the specifications of our microwave synthesizer. Furthermore, the fact that the direction of the phase noise is always tangential to the resonance circle (e.g. even at 


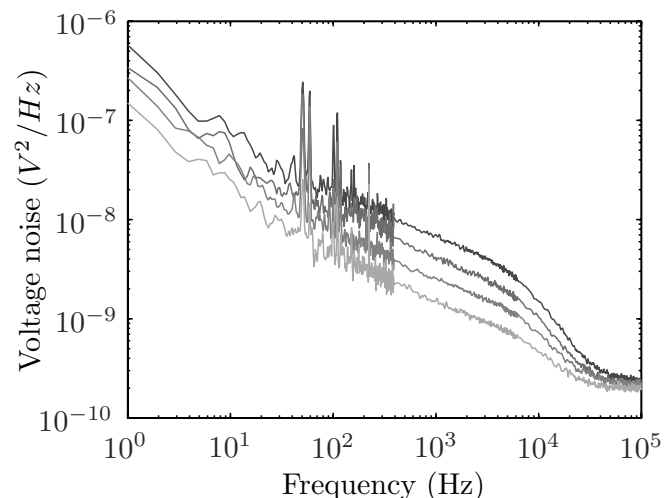

(a)

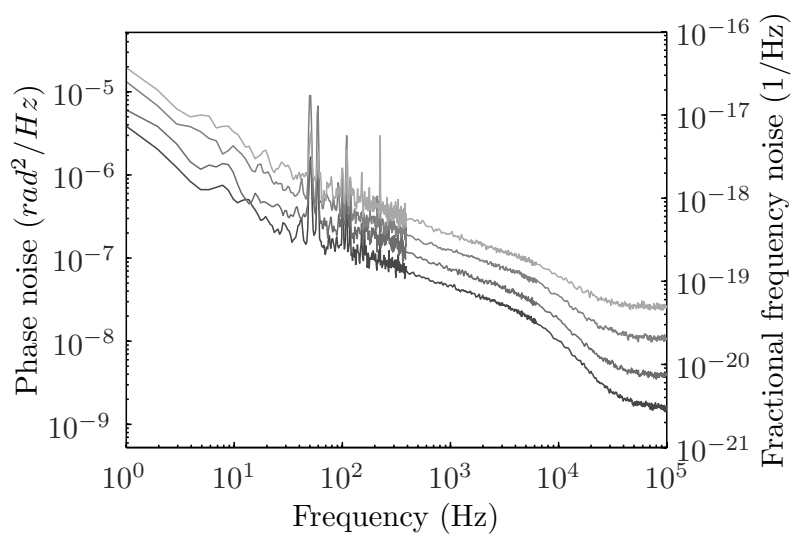

(b)

Figure 3. Spectra of excess noise in the phase direction at different readout powers. (a) Voltage noise spectrum $S_{\delta v}(\nu)$. (b) Phase noise spectrum $S_{\delta \theta}(\nu)$ (left axis) and fractional frequency noise spectrum $S_{\delta f_{0} / f_{0}}(\nu)$ (right axis). The readout powers of the 4 curves are $-87 \mathrm{dBm},-91 \mathrm{dBm},-95 \mathrm{dBm},-99 \mathrm{dBm}$ from top to bottom in (a) and from bottom to top in (b). The data is from a $200 \mathrm{~nm}$ thick $\mathrm{Al}$ on sapphire resonator.

points $\mathrm{A}$ and $\mathrm{B}$ ) indicates the noise is intrinsic to the resonator and rules out all noise sources in the readout system, including the synthesizer. This observation also rules out an explanation for the excess noise as being due to quasiparticle fluctuations, such as generation-recombination noise, excess radiation, temperature fluctuations, etc. Changes in the quasiparticle density would produce changes in both the inductance and resistance of the resonator, so the $I Q$ point would move along a trajectory that makes a nonzero angle with the resonance circle, as illustrated by the dashed curve in Figure 2(a). We conclude the excess noise is not related to quasiparticle fluctuations.

\subsection{Voltage noise, phase noise and frequency noise}

In a real measurement, the excess noise directly appears as a voltage noise: fluctuations in the voltage outputs of the IQ mixer (indicated by $\delta v$ in Figure 2(a)). Because the fluctuations are mostly in the tangential direction of the resonance circle, they contribute to a fluctuation in the measured angle (indicted by $\delta \theta$ ) but do not change the radius. Because the angle $\theta$ corresponds to the phase of the microwave excitation inside the resonator, the excess noise is essentially phase noise. This phase noise may be caused by many sources and most of them can be viewed as being equivalent to a jitter in the resonance frequency $f_{0}$. For example, temperature fluctuation will cause a fluctuation in the kinetic inductance and eventually a fluctuation in the phase velocity and $f_{0}$. Thus a frequency noise can be defined as the effective fluctuation in the resonance frequency $\delta f_{0}$ that would lead to $\delta \theta$. Frequency noise is useful in comparing the noise between two resonators that have different quality factors, because the same $\delta f_{0}$ leads to a larger $\delta \theta$ (and $\delta v$ ) for the resonator with a larger $Q$. To fairly compare noise among resonators with both different $f_{0}$ and different $Q$, we further define a fractional frequency noise as $\delta f_{0} / f_{0}$ (unitless).

Noise spectra in terms of voltage noise, phase noise, frequency noise and fractional frequency noise are related to each other by the following relationships:

$$
\begin{aligned}
& S_{\delta \theta}(\nu)=\frac{S_{\delta v}(\nu)}{r^{2}},\left(\delta \theta=\frac{\delta v}{r}\right) \\
& S_{\delta f_{0} / f_{0}}(\nu)=\frac{S_{\delta \theta}(\nu)}{16 Q^{2}},\left(\frac{\delta f_{0}}{f_{0}}=\frac{\delta \theta}{4 Q}\right) \\
& S_{\delta f_{0}}(\nu)=f_{0}^{2} S_{\delta f_{0} / f_{0}}(\nu),
\end{aligned}
$$




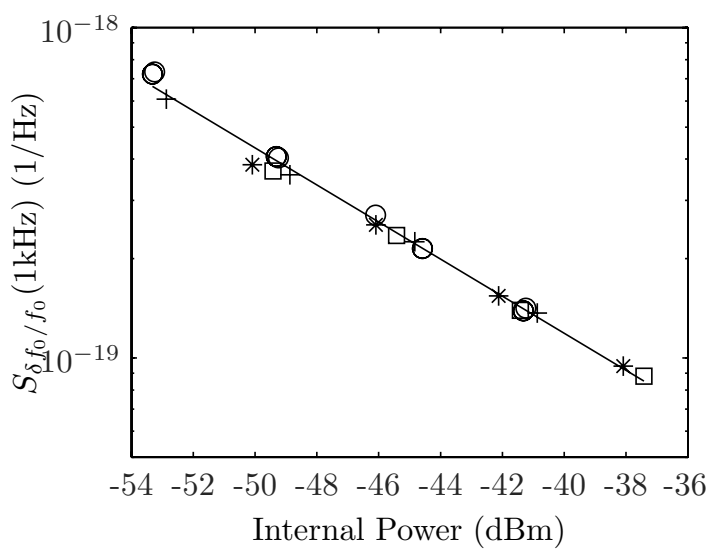

(a)

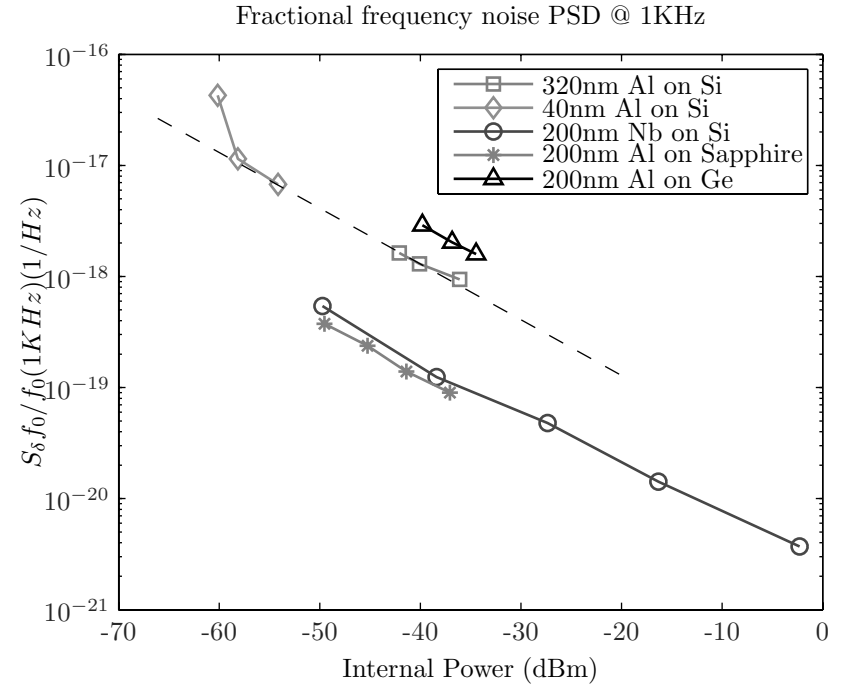

(b)

Figure 4. Fractional frequency noise PSD at $1 \mathrm{KHz}$ v.s. internal power falls on to straight lines of slope $-1 / 2$ in log-log plot indicating a power law dependance: $S_{\delta f_{0} / f_{0}} \propto P_{\text {int }}^{-1 / 2}$. (a) Fractional frequency noise of different resonators on the same substrate. Data points marked with "+", " $\square$ " and "**" are the noise of three different resonators (with different $f_{0}$ and $Q$ ) measured on-resonance at four different driving power. Data points marked with "o" are the noise of resonator-1 (marked with "*") measured at half-bandwidth away from the resonance frequency $\left(f_{0} \pm f_{0} / 2 Q\right)$ under the same 4 different driving power. The 3 resonators have the same thickness of $\mathrm{Al}(200 \mathrm{~nm})$ and are on the same sapphire substrate. (b) Comparison of fractional frequency noise measured from different metal-substrate combinations.

where $r$ is the radius of the resonance circle. They will be used to compare the excess noise in the following discussions.

\subsection{Power and Material Dependance}

One characteristic of this excess noise is its dependence on the power of the readout signal. Figure 3 compares the measured noise spectra of a resonator at four different readout powers in steps of $4 \mathrm{dBm}$. We found that the voltage noise increases with the readout power, as shown in Figure 3(a). Moreover, a $2 \mathrm{~dB}$ separation is found between two adjacent noise spectra, indicating that the voltage noise $\left(S_{\delta v}\right)$ is proportional to the square root of readout power $\left(P_{r}\right)$ :

$$
S_{\delta v}(\nu) \propto P_{r}^{\frac{1}{2}}
$$

On the contrary, when converted to phase noise or frequency noise, the noise is found to decrease with readout power. A same separation of $2 \mathrm{~dB}$ with a reversed order of the curves is seen in figure 3(b), indicating the relationship between the noise and the readout power is:

$$
S_{\delta \theta}(\nu) \propto P_{r}^{-\frac{1}{2}}, \quad S_{\delta f_{0} / f_{0}}(\nu) \propto P_{r}^{-\frac{1}{2}}
$$

Eqn. (5) and Eqn. (6) appear to be contradictory. They are consistent once we take account of the fact that the radius of the resonance loop $r$ also scales with the readout power:

$$
r \propto P_{r}^{\frac{1}{2}}
$$


We would also like to compare the excess noise among resonators with different $f_{0}$ and $Q$. To make fair comparisons, we plot the noise not against the readout power, but against the internal power of the resonator, which is defined as the power inside the resonator*. The fractional frequency noise against internal power of resonators with different $f_{0}$ and $Q$ on the same substrate are compared in Figure 4(a). The data points, from three different resonators, four different readout powers, driven on-resonance and detuned, all fall onto a straight line, suggesting that the fractional frequency noise only depends on the internal power $P_{\text {int }}$ (or the internal electric field $E_{\text {int }}$ ) of the resonator and by a power law

$$
S_{\delta f_{0} / f_{0}}(\nu) \propto P_{\text {int }}^{-\frac{1}{2}} \propto E_{\text {int }}^{-1} .
$$

We further investigated the excess noise on different substrate materials. Noise (fractional frequency noise spectrum density at $1 \mathrm{KHz}$ ) against internal power from five devices made of different metal-substrate combinations are compared in Figure 4(b). There we find two important features. First, the power law of Eqn.(8) is universal on all substrates, indicating a common noise source. Second, we find that the noise level are substrate dependent. In general, sapphire substrates give lower excess noise than Si or Ge substrates, with the one exception being the $\mathrm{Nb}$ on $\mathrm{Si}$ device ${ }^{\dagger}$. We notice that the two $\mathrm{Al}$ resonators with different thicknesses but on the same substrate $(\mathrm{Si})$ fall onto an equal-noise line in Figure 4(b), even though the kinetic inductance is much larger for the $40 \mathrm{~nm} \mathrm{Al} \mathrm{film.} \mathrm{These} \mathrm{results} \mathrm{strongly} \mathrm{suggest} \mathrm{the} \mathrm{substrate} \mathrm{is} \mathrm{responsible} \mathrm{for} \mathrm{the} \mathrm{excess} \mathrm{noise.}$

All the experimental results of power dependence of excess phase noise finally condense to a simple relationship of Eqn. (8). In fact, this relationship is more important than it appears to be. The power-law indices in Eqn. (8) are not trivial at all: Any additive noise (e.g., Johnson noise of the amplifiers) that does not depend on the amplitude of the signal will give a constant noise blob in the IQ plane and $S_{\delta f_{0} / f_{0}}(\nu) \propto P_{\text {int }}^{-1} \propto E_{\text {int }}^{-2}$ is expected instead of Eqn. (8). Similarly, any multiplicative noise (e.g., phase noise from the synthesizer) that is proportional to the amplitude of the signal will lead to a constant frequency noise $S_{\delta f_{0} / f_{0}}(\nu) \sim$ const. So Eqn. (8) is a physically interesting characteristic of the unknown noise source.

\section{DISCUSSION}

\subsection{Introduction to TLSs in amorphous solids}

It is well known that the low-temperature properties of amorphous solids can be remarkably different as compared to their crystalline counterparts. Indeed, over the past 30 years, the physical properties of amorphous solids have been explored in systematic studies, including measurements of specific heat, the variation of sound velocity, sound attenuation, dielectric dispersion and loss, and in some cases including nonlinear behavior. A two-levelsystem (TLS) model of the low-temperature behavior of amorphous solids was put forward by Phillips. ${ }^{15}$ This model assumes TLSs, with a wide range of excitation energies and relaxation rates, exist in these solids and interact with elastic or electromagnetic waves. Physically, a TLS is thought to consist of an atom or a group of atoms moving in a double-well potential. ${ }^{16}$ This model is very successful in explaining the observed phenomena and is generally accepted. We suggest that TLSs in the dielectric material of our resonators may be responsible for the excess phase noise.

\subsection{A potential explanation of the excess phase noise: fluorescence from TLSs}

A TLS excited by the standing wave in a microwave resonator with the microwave frequency close to the two-level transition is analogous to a two-level atom in an optical cavity driven at resonance by coherent light (laser). Such optical TLSs are well-studied in quantum optics and fully discussed in the resonance fluorescence theory. ${ }^{17 \ddagger}$

\footnotetext{
${ }^{*}$ Inside the resonator there is no traveling wave and the voltage has a standing wave pattern, $V_{0} \cos k z$, where $V_{0}$ is the maximum voltage. We define $P_{i n t}=\frac{V_{0}^{2}}{2 Z_{0}}$, where $Z_{0}$ is the characteristic impedance.

${ }^{\dagger}$ We do not have an explanation for this yet. It is possible that the etching chemistry in the fabrication process also plays a role.

${ }^{\ddagger}$ There is a difference between the two cases on the relaxation mechanism. In optical resonance, the relaxation of a two-level atom is through spontaneous emission into a photon bath. Because the photon energy $h \nu$ is much larger than $k_{b} T$, the bath is not thermally excited and has only vacuum modes. In our case, the relaxation of a TLS is mainly through coupling to a phonon bath. Because the phonon energy $h \nu$ is comparable to $k_{b} T$, the bath can be thermally excited. Thus A temperature dependent relaxation rate is expected in our case.
} 


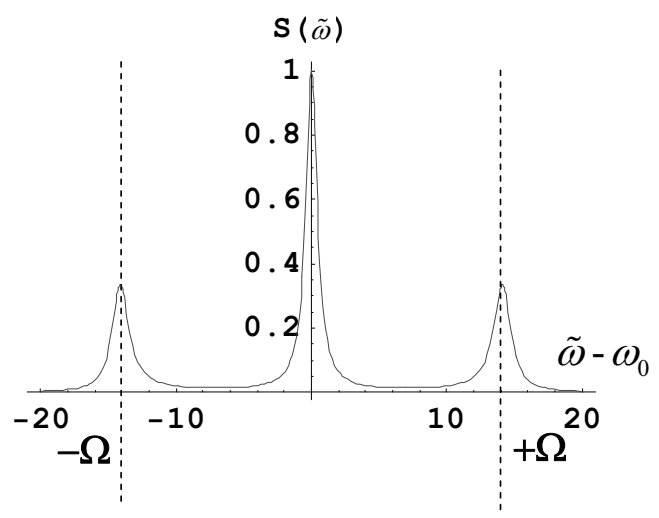

Figure 5. Fluorescence spectrum $S(\widetilde{\omega})$ from a TLS driven exactly on resonance $\left(\omega=\omega_{0}\right)$, where $\widetilde{\omega}, \omega$ and $\omega_{0}$ are the frequency of the fluorescent light, the frequency of the driving field, and the frequency of the two-level transition respectively. The plot is made using $\kappa=1$ and $\Omega=14$

If the excess noise were due to the fluorescence emitted by the TLSs, we would expect the noise spectrum to be closely related to the fluorescence spectrum. We find ourselves in the strong driving regime where the Rabi frequency $(\Omega$, estimated to be above $30 \mathrm{MHz})$ is much greater than the relaxation rate $(\kappa$, estimated to be below $1 \mathrm{MHz})$. A typical fluorescence spectrum in this regime is shown in Figure. 5, which is known as the famous Mallow triplet. ${ }^{18}$ It consists of three Lorentzian peaks: the central peak with a width of $\kappa / 2$ at the driving frequency and two side peaks with widths of $3 \kappa / 2$ separated from the central peak by the Rabi frequency $\Omega$.

The resonance fluorescence theory further predicts that the central peak of the Mallow triplet corresponds to the in-phase component of the fluorescence spectrum whereas the side peaks correspond to the quadrature-phase component. It can be shown that in our setup the in-phase component and quadrature-phase component of the fluorescence will be converted to the phase noise and the amplitude noise respectively. Because our resonators act as band-pass filters centered at $\omega$ with bandwidths (tens of $\mathrm{kHz}$ ) less than the Rabi frequency, they select out only the central peak and filter away the two side peaks. This may explain why we observe excess phase noise rather than amplitude noise.

The above discussion is quite qualitative and more details on the resonance fluorescence can be found in any quantum optics book. ${ }^{17}$ Another paper discussing phase noise with more theoretical details will be coming soon.

\section{CONCLUSION}

Excess noise in MKIDs is studied. The excess noise appears purely as phase noise and no amplitude noise in excess is observed. We found that the excess phase noise has a power law dependence on the internal power of the resonator. The power-law index is found to be $-1 / 2$, independent of the superconductor and substrate. The noise level, however, strongly depends on the substrate. We suggest that the excess noise may be explained by the fluorescence from the TLSs in the dielectric material of the resonator. Applying the resonance fluorescence theory and two-level-system model may lead to a full understanding of the excess phase noise.

\section{REFERENCES}

1. P. K. Day, H. G. LeDuc, B. A. Mazin, A. Vayonakis, and J. Zmuidzinas, "A broadband superconducting detector suitable for use in large arrays," Nature 425(6960), pp. 817-821, 2003.

2. B. A. Mazin, P. K. Day, J. Zmuidzinas, and H. G. LeDuc, "Multiplexable kinetic inductance detectors," in Ninth International Workshop on Low Temperature Detectors, F. S. Porter, D. McCammon, M. Galeazzi, and C. K. Stahle, eds., AIP Conf. Proc. 605, pp. 309-312, New York: AIP, 2002.

3. B. A. Mazin, P. K. Day, H. G. Leduc, A. Vayonakis, and J. Zmuidzinas, "Superconducting kinetic inductance photon detectors," SPIE Proc. 4849, pp. 283-293, Dec. 2002. 
4. J. Zmuidzinas, B. Mazin, A. Vayonakis, P. Day, and H. G. LeDuc, "Multiplexable kinetic inductance detectors," in Proceedings far-IR, sub-mm, and mm detector workshop, J. Wolf, J. Farhoomand, and C. R. McCreight, eds., NASA/CP-2003-211408, pp. 326-329, Washington, DC: NASA, 2003.

5. G. A. Vardulakis, S. Withington, and D. J. Goldie, "Theoretical modeling of optical and x-ray photon counting kinetic inductance detectors," SPIE Proc. 5499, pp. 348-359, Sept. 2004.

6. A. Porch, P. M. abd S. Doyle, and C. Dunbscombe, "Calculation of the characteristics of coplanar resonators for kinetic inductance detectors," IEEE Trans. Appl. Supercond. 15, pp. 552-555, June 2005.

7. B. A. Mazin, P. K. Day, K. D. Irwin, C. D. Reintsema, and J. Zmuidzinas, "Digital readouts for large microwave low-temperature detector arrays," Nuclear Instruments and Methods in Physics Research A 559, pp. 799-801, Apr. 2006.

8. P. K. Day, H. G. Leduc, A. Goldin, T. Vayonakis, B. A. Mazin, S. Kumar, J. Gao, and J. Zmuidzinas, "Antenna-coupled microwave kinetic inductance detectors," Nuclear Instruments and Methods in Physics Research A 559, pp. 561-563, Apr. 2006.

9. J. Gao, J. Zmuidzinas, B. A. Mazin, P. K. Day, and H. G. Leduc, "Experimental study of the kinetic inductance fraction of superconducting coplanar waveguide," Nuclear Instruments and Methods in Physics Research A 559, pp. 585-587, Apr. 2006.

10. J. J. A. Baselmans, R. Barends, J. N. Hovenier, J. R. Gao, H. Hoevers, and T. M. Klapwijk, "Development of high- $Q$ superconducting resonators for use as kinetic inductance sensing elements," Nuclear Instruments and Methods in Physics Research A 559, pp. 567-569, Apr. 2006.

11. K. D. Irwin, "An application of electrothermal feedback for high resolution cryogenic particle detection," App. Phys. Lett. 66, pp. 1998-2000, 1995.

12. N. E. Booth and D. J. Goldie, "Superconducting particle detectors," Superconductor Science and Technology 9(7), pp. 493-516, 1996.

13. B. A. Mazin, Microwave kinetic inductance detectors. PhD thesis, California Institute of Technology, 2004.

14. D. C. Mattis and J. Bardeen, "Theory of the anomalous skin effect in normal and superconducting metals," Phys. Rev. 111, pp. 412-417, 1958.

15. W. A. Phillips, "Tunneling states in amorphous solids," Journal of Low Temperature Physics 7, pp. 351-360, 1972.

16. J. Reinisch and A. Heuer, "What is moving in silica at $1 \mathrm{~K}$ ? A computer study of the low-temperature anomalies," Phys. Rev. Lett. 95, p. art. 155502, Oct. 2005.

17. D. Walls and G. Milburn, Quantum optics, Springer-Verlag, Berlin; New York, 1994.

18. B. R. Mollow, "Power spectrum of light scattered by two-level systems," Phys. Rev. 188, pp. 1969-1975, 1969. 